\title{
La traducción literal en el ámbito audiovisual. Método y técnica
}

\section{Literal translation in the audiovisual field. Methods and techniques}

\section{Juan José MARTíNEZ SIERRA}

Universitat de València

Recibido: 10-2-2016. Revisado: 4-8-2016. Aceptado: 28-2-2017.

Resumen: El debate alrededor de la traducción literal viene de lejos y, seguramente, nos seguirá acompañando durante mucho más tiempo. En todo caso, no se pretende aquí abordar dicha discusión, sino más bien realizar una mirada descriptiva y no valorativa del asunto, con el único propósito de señalar la necesidad de una mayor precisión terminológica cuando de hablar de traducción literal se trata, especialmente en el aula de traducción. Como se detallará, la traducción literal puede ser tanto un método como una técnica de traducción, y parece conveniente ser conscientes de a qué nos estamos refiriendo exactamente cuando hacemos uso de esta etiqueta, con objeto de evitar confusiones. Por otro lado, como se mostrará, las posturas en uno u otro sentido (método o técnica) siguen vigentes, si bien los datos sugieren que, pese a lo que podría parecer en un principio, la traducción literal, como método, está seguramente más presente en la práctica de la traducción audiovisual de lo que se pueda pensar.

Palabras clave: traducción literal; traducción audiovisual; método; técnica; metalenguaje. 


\begin{abstract}
The debate on literal translation is an old one, and it will surely continue to exist for the time to come. In any case, it is not intended to address that discussion here, but rather to take a descriptive, nonjudgmental look at the matter for the sole purpose of pointing out the need for more precise terminology when speaking about literal translation, especially in translation teaching. As it will be explained, literal translation can be both a translation method and a translation technique and it seems appropriate to be aware of what we mean exactly when we use this label. Moreover, as it will be shown, the positions in either direction (method or technique) remain in force, although the data suggest that, despite what it might seem at first, literal translation, as a method, is probably more present in the practice of audiovisual translation than what one might think.
\end{abstract}

Keywords: literal translation; audiovisual translation; method; technique; metalanguage.

\title{
1. INTRODUCCIÓN
}

¿Qué es la traducción literal? Dejando a un lado, de momento, la literatura al respecto, la experiencia docente nos demuestra que existe cierta confusión al respecto. Nuestros estudiantes universitarios, expuestos a multitud de inputs provenientes de diversos ámbitos (Internet, ciertas publicaciones, afirmaciones erradas realizadas por pseudotraductólogos...), en ocasiones asocian la expresión traducción literal a soluciones como las que a continuación se ofrecen, recogidas por Torregrosa (2012, 91):

$>$ Let's make a toast - Hagamos una tostada.

$>$ Chin up! - ¡Barbilla arriba!

$>$ l'd like the whole family checked - Quiero a toda la familia a cuadros.

$>$ Piss off - Méate fuera.

$>$ Human guinea pigs - Cerdos guineanos humanos.

Efectivamente, lo anterior es traducción literal ${ }^{1}$, pero también lo es lo siguiente:

$>$ My computer needs new software - Mi ordenador necesita un nuevo software.

$>$ I love science fiction films - Me encantan las películas de ciencia ficción.

$>$ The boy eats an apple - El chico come una manzana.

1. Una técnica literal incorrectamente usada puede ser fuente de un error de traducción, si bien ese es un escenario que no vamos a desarrollar en el presente trabajo y cuyo tratamiento reservamos para futuras contribuciones.

Juan José MARTÍNEZ SIERRA La traducción literal en el ámbito audiovisual. Método y técnica 
$>$ He takes a walk every day - Él da un paseo todos los días².

$>$ Houston, we have a problem - Houston, tenemos un problema.

$>$ It's raining cats and dogs - Está lloviendo a cántaros.

¿Cuál es entonces la diferencia? Precisamente, el objetivo de este trabajo es tratar de arrojar luz sobre este asunto, con objeto de definir qué es exactamente la traducción literal y cómo se puede abordar en el aula. No es nuestro propósito, por tanto, defender o atacar el uso de la traducción literal como método o como técnica, sino más bien abogar por una claridad terminológica al respecto. Haremos todo esto en el ámbito de la traducción audiovisual (si bien lo postulado podría seguramente ser extensible, en mayor o menor medida, a la traducción en general), en el que esta cuestión es especialmente relevante (baste recordar lo que ocurre en el caso de la traducción para doblaje o para subtitulación, dos prácticas en las que la alusión al fenómeno de la traducción literal es frecuente) y en cuya docencia nos vemos, en no pocas ocasiones, ante la necesidad de eliminar prejuicios al respecto y defender las virtudes, que también las tiene, y de acotar los defectos de este proceder traductológico. Para conseguir nuestros fines, recurriremos a distintas posturas adoptadas desde diferentes esferas.

\section{LAS TÉCNICAS DE TRADUCCIÓN}

Por técnica de traducción podemos entender el «[p]rocedimiento, visible en el resultado de la traducción, que se utiliza para conseguir la equivalencia traductora a microunidades textuales» (Hurtado 2001, 642). A esto podemos añadir que, «[b]ásicamente, se trata de etiquetar una solución específica para un problema muy concreto (y microtextual) del encargo de traducción» (Martí Ferriol 2010, 91). Por tanto, «[a] diferencia del método, que es una opción global que recorre todo el texto y que afecta al proceso y al resultado, la técnica afecta sólo al resultado y a unidades menores del texto" (Hurtado 2001, 257).

Martí Ferriol $(2006,73)$ coincide con Marco $(2004,130)$ en que el concepto de técnica es de crucial importancia en Traductología y señala que «aunque haya sido denostado por algunos autores, posiblemente por su forma de entenderlo en un sentido prescriptivo, [...] es fundamental en cualquier trabajo de investigación descriptivo". Anteriormente, Hurtado se pronuncia sobre este particular en líneas similares (2001, 257):

2. Este ejemplo está tomado de Newmark $(1988,101)$. Nuestro agradecimiento a José Luis Martí Ferriol por ponernos sobre su pista.

Juan José MARTÍNEZ SIERRA La traducción literal en el ámbito audiovisual. Método y técnica vol. 3-1, June 2017, 13-34

elSSN: 2444-1961

(c) Ediciones Universidad de Salamanca - CC BY-NC-ND 
Como consecuencia del carácter prescriptivo que ha prevalecido en torno a las técnicas de traducción, esta noción ha sido desechada por algunos teóricos. Por nuestra parte, pensamos que el interés mayor de las técnicas de traducción radica en el hecho de que proporcionan un metalenguaje y una catalogación que sirve para identificar y caracterizar el resultado de la equivalencia traductora con respecto al texto original. Por consiguiente, sirven como instrumento de análisis para la descripción y comparación de traducciones, al lado de las categorías textuales [...], contextuales [...] y procesuales [...]. Las técnicas de traducción permiten identificar, clasificar y denominar las equivalencias elegidas por el traductor para microunidades textuales así como obtener datos concretos sobre la opción metodológica utilizada, pero, evidentemente, no bastan por sí solas como instrumento de análisis.

No se trata, sin embargo, de un término libre de confusiones. Hurtado (2001, 264-266) pone sobre la mesa diversos desacuerdos terminológicos relacionados con la denominación de las técnicas de traducción: procedimiento, estrategia, error o técnica de ajuste. No es nuestro deseo ni objetivo detenernos en este debate y nos limitaremos, por tanto, a manifestar nuestro acuerdo con la autora cuando señala la conveniencia de distinguir entre método, técnica y estrategia (Hurtado 2001, 256). Aquí nos ocupamos de los dos primeros conceptos.

\section{LA TRADUCCIÓN LITERAL COMO TÉCNICA DE TRADUCCIÓN}

Hurtado $(2001,257)$ realiza un completo repaso de las distintas definiciones y clasificaciones propuestas de las técnicas de traducción, y remitimos al lector a dichas páginas para así obtener un retrato de lo expuesto por autores como Vinay y Darbelnet (1958), Nida (1964), Vázquez Ayora (1977), Delisle (1993) o Newmark (1988). Este repaso es también recogido por Martí Ferriol (2010, 92-94), quien da un paso adelante y formula una propuesta de técnicas capaces de dar cuenta del ámbito audiovisual: préstamo, calco, traducción palabra por palabra, traducción uno por uno, traducción literal, equivalente acuñado, omisión, reducción, comprensión, particularización, generalización, transposición, descripción, ampliación, amplificación, modulación, variación, substitución, adaptación y creación discursiva.

No es nuestra intención entrar a valorar en detalle la propuesta de Martí Ferriol arriba recogida. Nos interesa más el hecho de que la disposición numerada de las diferentes técnicas no es casual, sino que se sitúan en un continuo que obedece a un criterio que tiene como resultado la distribución en tres bloques de todas ellas, tal y como se observa en la siguiente figura:

Juan José MARTÍNEZ SIERRA La traducción literal en el ámbito audiovisual. Método y técnica 


\section{CLASIFICACIÓN DE TÉCNICAS DE TRADUCCIÓN SEGÚN EL MÉTODO DE TRADUCCIÓN}

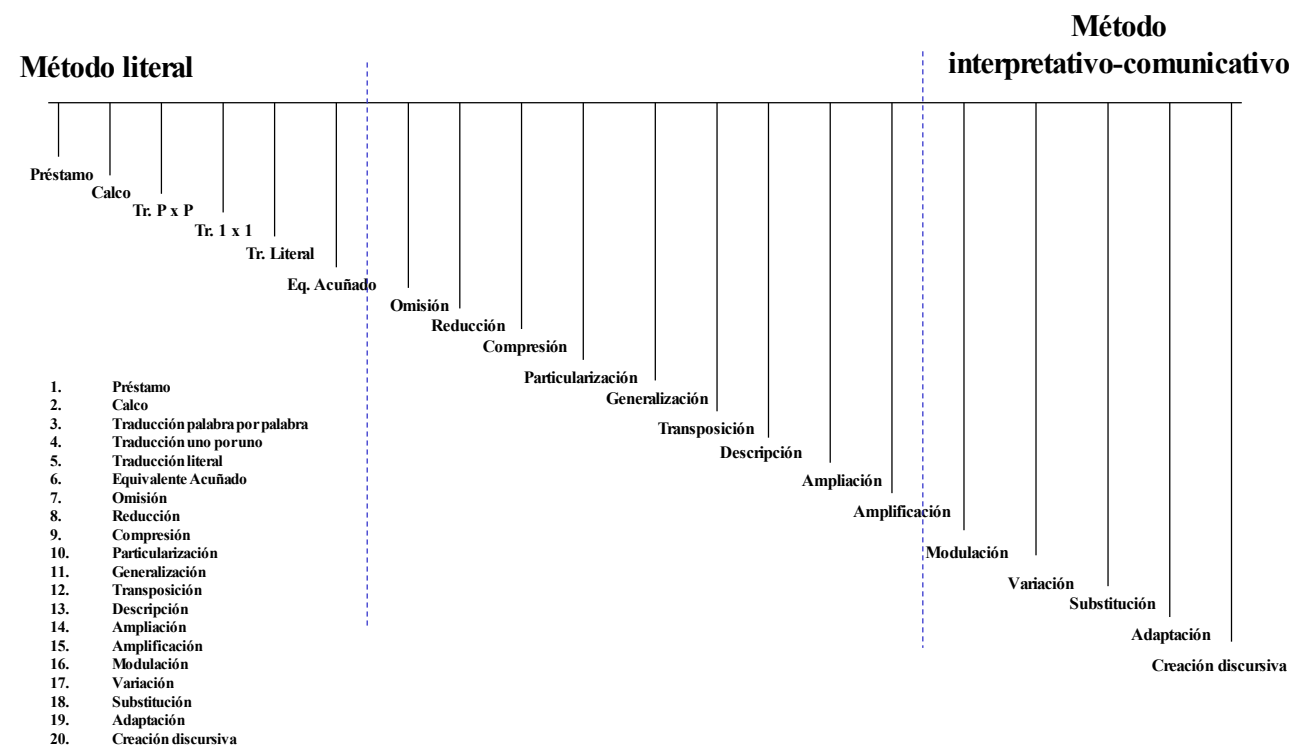

Figura 1. Técnicas de traducción según el método de traducción (Martí Ferriol 2013, 122).

Por un lado, Martí Ferriol identifica un grupo de técnicas próximas a la extranjerización. Por otro, un grupo cercano a la familiarización. Finalmente, el autor habla de una zona intermedia (2013, 122-123):

Las técnicas numeradas del 1 al 6 son más literales: desde la incorporación del préstamo, la copia del calco y la gradación en los tres tipos de traducciones literales, se llega al equivalente acuñado, donde se situaría el límite de la literalidad rigurosa. [...] Por el lado derecho (las técnicas numeradas del 16 al 20), se aprecia un grado creciente de familiarización: desde la modulación (ese cambio del punto de vista que se muestra tan versátil), se observa una evolución del grado de implicación en la creatividad del traductor, hasta llegar a la creación discursiva, paradigma de la creación ad hoc para cualquier tipo de problema. [...] La zona intermedia (técnicas numeradas del 7 al 15) corresponde a la de las argucias traductoras de carácter más lingüístico. Existe también una gradación lógica en esta zona intermedia. En el lado izquierdo de esta zona (7 a 10) se identifican las técnicas que suprimen o eliminan elementos lingüísticos. En el lado derecho de esta zona (11 a 15) se encuentran las técnicas que los modifican o amplían. 
La palabra literal aparece en dos ocasiones: como técnica (la número 5)³ y como método (el literal, equiparable, por otro lado, a la extranjerización de Venuti 1995 o a la adecuación de Toury 1995). Veamos a continuación qué podemos entender por método.

\section{EL MÉTODO DE TRADUCCIÓN}

Según Hurtado (2001, 639), un método se puede definir como el «[d]esarrollo de un proceso traductor determinado regulado por unos principios en función del objetivo del traductor, respondiendo a una opción global que recorre todo el texto». De manera similar, Martí Ferriol $(2010,94)$ lo define como «el resultado del empleo, consciente o inconsciente, de una serie de normas y técnicas de traducción, que configuran la opción metodológica escogida por el traductor».

Hurtado $(2001,639)$ añade que «[l]os métodos cambian en función del contexto y de la finalidad de la traducción». Esto está en consonancia con lo expresado por Martí Ferriol $(2006,76)$, cuando dice que «el empleo de un método de traducción implica el uso de unas técnicas determinadas y viceversa" y que "[d]ebe entenderse esta relación en un sentido general, o macrotextual».

\section{LA TRADUCCIÓN LITERAL COMO MÉTODO Y TÉCNICA DE TRADUCCIÓN: EL ORIGEN DE LA CONFUSIÓN}

Hurtado $(2001,252)$ identifica cuatro métodos básicos: el interpretativocomunicativo, el literal, el libre y el filológico. Si nos centramos en el segundo, al que también califica de traducción literal, la autora lo define como el «[m]étodo traductor que se centra en la reconversión de los elementos lingüísticos del texto original, traduciendo palabra por palabra, sintagma por sintagma o frase por frase, la morfología, la sintaxis y/o la significación del texto original». A esto, Hurtado $(2001,639)$ añade que «[h]ay que distinguir entre método literal, que afecta a todo el texto, y técnica de traducción literal, que afecta a microunidades textuales", y es aquí donde probablemente reside buena parte de las concepciones erróneas que se pueden dar cuando se habla de traducción literal, puesto que en no pocas ocasiones se confunde la parte con el todo.

Mayoral (2013, en línea) ya parece pronunciarse en este sentido cuando comenta que «podemos advertir [...] un gran obstáculo en nuestra discusión; estamos mezclando

3. Recordemos que Martí Ferriol (2013) habla de tres tipos de traducciones literales: traducción palabra por palabra, traducción uno por uno y traducción literal. 
distintos procedimientos: estrategias de traducción, que afectan a todo el texto, con técnicas de traducción, que intentan resolver problemas puntuales».

En esta línea se pronuncia también Martí Ferriol $(2006,285)$, cuando se propone como uno de sus objetivos «[p]rofundizar en el concepto de 'traducción literal', intentando poner de manifiesto que a lo largo de la historia de la Traductología se ha incluido bajo esta denominación diferentes conceptos no totalmente idénticos entre sí». El autor representa gráficamente la distinción de la siguiente manera:

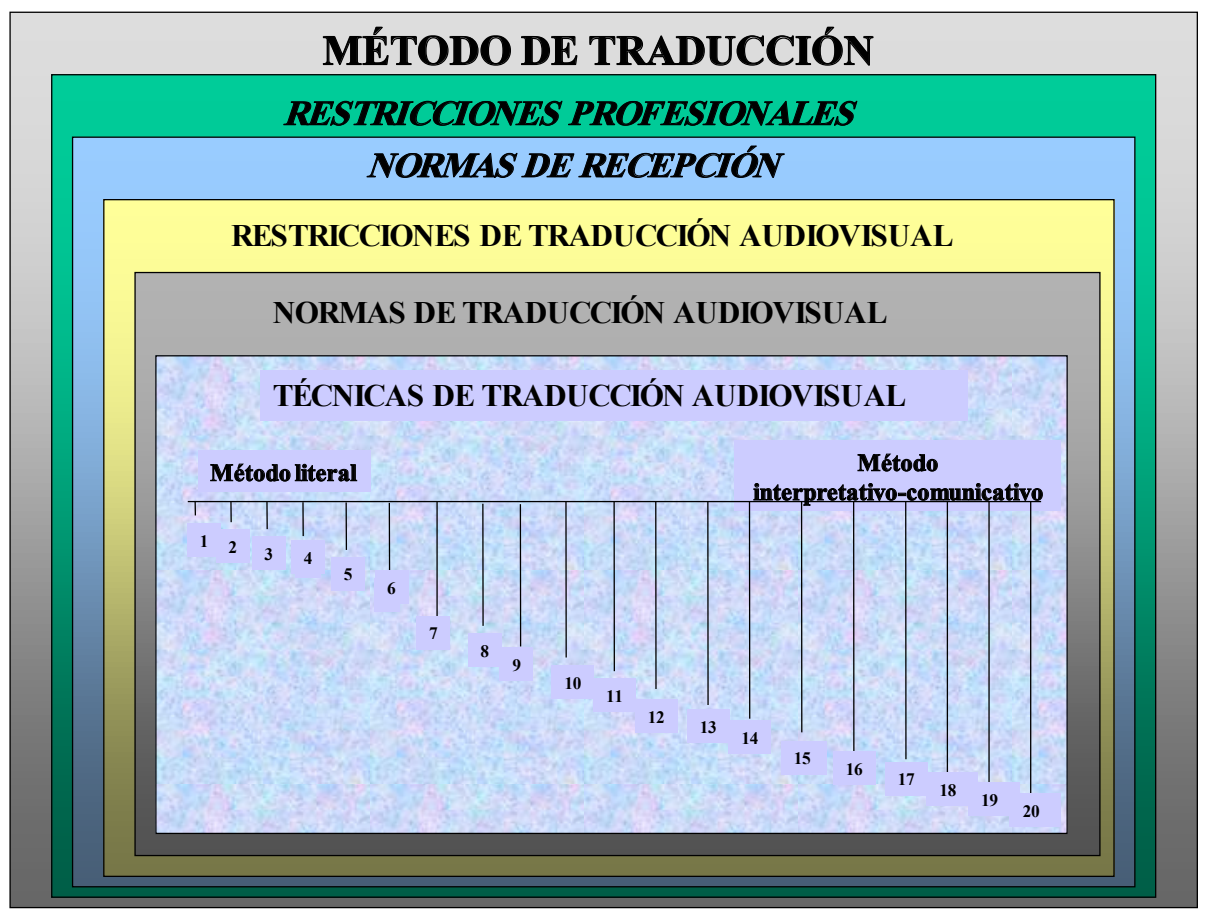

Figura 2. Representación gráfica del método de traducción (Martí Ferriol 2006, 148).

\section{LA TRADUCCIÓN LITERAL: UNA DOBLE LECTURA}

Por tanto, cuando leemos argumentos en pro o en contra de la traducción literal, ¿a qué se refieren exactamente? ¿Hablan todos de lo mismo? A tenor de lo que una revisión, siquiera breve, de la bibliografía indica, la respuesta parece ser negativa, puesto que, salvo en alguna excepción, se habla de traducción literal sin precisar exactamente a qué se refieren. Nos centraremos en dos casos, el del doblaje y el de la subtitulación, por ser estas las modalidades de traducción audiovisual que, seguramente, mayor debate han generado en torno al tema que estamos discutiendo. 


\subsection{El doblaje}

Chaves $(2000,113)$ expresa que, en el doblaje, la traducción «[n]o puede ser una simple transposición literal de una lengua a otra», aludiendo a las diferentes densidades de las lenguas o a las "diferentes formas de hablar» que las situaciones dramáticas originan.

En líneas similares se pronuncian los alumnos del profesor Castillo Flores (2011, en línea) en su blog sobre traducción, cuando indican que «[n]i la subtitulación ni el doblaje permiten una traducción literal» y que «no siempre podemos traducir literalmente».

Podemos asimismo mencionar a Sorroche (2010, en línea) quien, desde el ámbito de los videojuegos, comenta que «las pruebas no son fragmentos al azar. Suelen contener juegos de palabras, giros, expresiones y referencias culturales. En estos casos, hay que hacer gala de imaginación y de creatividad por encima de todo. No nos limitemos a utilizar una traducción literal».

Como hemos visto, una mínima mirada a lo escrito sobre esta cuestión pone de manifiesto que existen voces que abogan por la no literalidad de la traducción, si bien no se acaba de precisar qué se entiende exactamente por traducción literal. Ahora bien, dicha revisión de lo escrito al respecto también deja aflorar argumentos que van en la dirección contraria, aunque de nuevo haciendo un uso genérico de la etiqueta traducción literal.

Si seguimos centrados en el caso del doblaje, en páginas como Laboris.net (2014, en línea) podemos leer que «[e]l proceso de doblaje se inicia con la traducción literal del texto original por parte de un traductor y su posterior adaptación».

De manera similar, Freijo y Torre (2014, en línea) escriben en Eldoblaje.com que «[e]l adaptador comienza su trabajo basándose en la traducción literal de los diálogos» (una rough translation, en términos de Whitman 1992), y que «[a]ctualmente, el pautado se hace digitalmente en la mayoría de los casos, pero no siempre, y no se utiliza el guión original, como se hacía antes, sino una traducción literal o un libro de diálogos ya adaptados en el idioma del doblaje». Ahora bien, hemos de decir que se percibe una cierta contradicción cuando Freijo y Torre añaden otras ideas como que «[l]a traducción es uno de los pilares principales sobre los que descansa un doblaje. Debe ser lo más fiel posible al idioma original, pero no excesivamente literal». Aquí cabría, además, preguntarse ¿qué significa «no excesivamente literal»? ¿Según qué escala? En cualquier caso, es posible encontrar pronunciamientos de esta índole en otros trabajos.

Cómitre (2009, en línea), por ejemplo, en su estudio sobre la traducción para doblaje de spots, argumenta que, en la fase de traducción, «[e]l traductor suele realizar una traducción literal del texto original con la que pretende transmitir el contenido semántico al director creativo que valora la pertinencia de realizar modificaciones en la 
parte visual del spot». La autora recoge también que otros autores como Martín (1994, 325) también hablan de la traducción literal como la tarea del traductor.

En este sentido se pronuncia también Rioz (2010, 135), cuando comenta que «[e]n el doblaje, el traductor no debe permitirse tanta pérdida de información, y puede realizar una traducción más 'literal' del guión original». Simón $(2008,22)$, directora, actriz y adaptadora de doblaje, va quizás un poco más allá, y señala que «la traducción de María José es muy buena, pero su deber es hacer una traducción literal»4.

En lo que se refiere al ajuste, encontramos argumentos parecidos en Kahane (1990-91, 117), quien escribe que «[l]a labor del traductor de doblaje es simplemente una transposición literal de un idioma a otro, sin preocuparse de los sincronismos, de la pronunciación o del movimiento de los labios de los actores». Esta última idea es criticada por Chaves $(2000,97)$, quien aduce que «durante mucho tiempo esto ha sido así, incluso lo es ahora [...], de ahí la pésima calidad de muchos doblajes que hemos podido ver».

Martínez Tejerina $(2012,176)$ obtiene resultados en este sentido y los justifica del siguiente modo: «el público actual sabe que esta comedia es de los Hermanos Marx y la idea generalizada en España hoy en día es que estos cómicos cultivan el humor del absurdo. Tales conocimientos fomentan la aceptación de un texto cargado de incongruencias y, en consecuencia, favorecen el éxito de la traducción literal como fuente de absurdos».

Chaves $(2000,96)$ encuentra una explicación a este tipo de actuaciones que escapa al puro ámbito traductológico: "cuando nos referimos al doblaje, también estamos hablando de una actividad económica, de una empresa, y en términos de rapidez y rentabilidad y, como es habitual, 'el cliente siempre tiene razón'». La autora alude a Delmas (1977), e interpreta sus palabras del siguiente modo (2000, 97): «es un error intentar hacer gala de virtuosismo a nivel léxico o sintáctico [y] no hay que intentar dar color al texto o buscar innovaciones de vocabulario». Chaves opina que este tipo de afirmaciones pueden parecer extrañas, si bien «de lo contrario es precisamente de lo que se quejan a menudo los directores de doblaje», a lo que añade que «la mayoría de los traductores, acostumbrados a hacer traducciones de novelas o de textos escritos, intentan, cuando abordan un guión, buscar equivalentes perfectos y los giros más adecuados, cuando lo que se precisa no es eso» (2000, 96, el énfasis es nuestro).

Resulta relevante, por último, mencionar que en el programa de estudios de alguna escuela de doblaje, como la de Barcelona, se incluye la Traducción literal como una asignatura más, junto a otras como Aspectos históricos del doblaje, Adaptación de guion y ajuste o La voz y el micrófono.

4. La autora se refiere a María José Aguirre, traductora de la serie Los Simpson para el mercado español.

Juan José MARTÍNEZ SIERRA La traducción literal en el ámbito audiovisual. Método y técnica 


\subsection{La subtitulación}

También es posible hallar pronunciamientos similares, en ambos sentidos, desde el ámbito de la subtitulación. Díaz Cintas y Remael $(2007,56)$, por ejemplo, se refieren a la importancia de respetar cuestiones como la sintaxis del original y de conseguir la máxima sincronía, argumentando, entre otras cosas, que no facilita la comprensión el hecho de escuchar un texto y de leer otro distinto de manera simultánea, idea que recogen de Ivarsson y Carroll $(1998,73)$. Díaz Cintas y Remael $(2007,57)$ señalan el aumento de la posible crítica a este respecto cuando la lengua origen es el inglés o una lengua con raíces lingüísticas similares a la meta. En alusión a los receptores, señalan que éstos «may feel cheated when the aggressive or rude performance of an actor leads them to expect a certain type of vocabulary that is not relayed in the translation, when a laconic exchange becomes a lengthy subtitle, or when an actor who speaks in linguistic waterfall is given in very brief subtitles». Ahora bien, por otro lado, concluyen que existe un límite respecto a hasta dónde podemos llegar siendo fieles al texto origen, no sólo por las restricciones técnicas ${ }^{5}$, sino también porque "the target language cannot be infinitely stretched». Aluden a Kriek (2002) y a su concepto de halve vertalingen (half-way translations), para los subtítulos que presentan, de manera casi literal, expresiones o frases del texto origen. Díaz Cintas y Remael se muestran contrarios a este proceder, cuando sentencian que "[t]his is certainly not an example to be emulated", si bien reconocen que «DVD subtitles tend to stay closer to the ST language than subtitles for television or the cinema» (2007, 57). ¿Podemos entender que por closer los autores quieren decir literales? De ser así, y dejando de lado los pronunciamientos a favor o en contra de la traducción literal, esta última afirmación resulta interesante, ya que evidencia que el tipo de soporte puede ser también decisivo a la hora de optar por un método.

No sólo el soporte puede influir, sino también el tipo de espectador meta o, como veremos, el propósito de los subtítulos. Respecto a la primera consideración, en su trabajo sobre el subtitulado para sordos, Pereira, refiriéndose al subtitulado interlingüístico para personas no sordas, explica que, en este tipo de trasvase, «el texto va acompañado del material lingüístico original». De este modo, dicho texto "se ve sometido a la evaluación y crítica de los espectadores, sepan estos el idioma original o no, hecho que repercute en la práctica del mediador al que, cada vez más, se le solicitan traducciones 'literales' lo menos alejadas posible del original». Sin embargo, la autora señala igualmente que «no debemos olvidar que gran parte de los espectadores sordos leen los labios, así que en la práctica también someten a escrutinio los subtítulos a ellos dirigidos y se quejan de que en aquellos no se lee lo

5. En este sentido, Botella Tejera (2007, en línea) señala que, en el caso de la subtitulación, sus «características propias [...], entre las que destaca la condensación, [...] no permitirán una traducción literal, ni una fidelidad absoluta al texto original».

Juan José MARTINEZ SIERRA La traducción literal en el ámbito audiovisual. Método y técnica
CLINA

vol. 3-1, June 2017, 13-34

elSSN: 2444-1961

(C) Ediciones Universidad de Salamanca - CC BY-NC-ND 
que, en ocasiones, descifran de los labios de los personajes». De este modo, «Al igual que los espectadores oyentes, también piden subtítulos literales y, en ambos casos, creemos que las críticas responden a la falta de conocimiento de las limitaciones que condicionan este tipo de trasvase» $(2005,162)$.

Mayoral $(2001,40)$ también considera este efecto cotilla cuando expresa que «[e]l espectador que comprende la lengua de los diálogos originales y la lengua de los subtítulos está constante e inevitablemente comparando ambas versiones. Y esta comparación la hace siguiendo sus propios criterios sobre lo que es o deja de ser una traducción acertada». En esta misma línea, el autor (2001, 40-41) añade:

[... p para aquellos espectadores poco o nada familiarizados con la traducción profesional, la traducción más adecuada sigue siendo la traducción más literal, entendiendo aquí como traducción más literal la que traduce íntegramente, sin añadir ni quitar nada, la que ofrece los significados en el mismo orden que el original, y la que rinde los significados más habituales de las palabras en los diccionarios. Esto conduce a lo siguiente: evitar la insatisfacción del espectador familiarizado con la lengua extranjera con los subtítulos, pues siempre que oiga 'turkey' esperará leer 'pavo'.

Como antes se avanzaba, el propósito de los subtítulos puede jugar también algún papel en todo este océano de decisiones. Como explica Díaz Cintas, muchas personas hacen uso del subtitulado para sordos (intralingüístico) para aprender otra lengua. De hecho, existen empresas que han sabido ver ahí un mercado. Ya en la década de 1990 Columbia Tristar lanzó la colección de vídeos SpeakUp, una serie de películas en lengua inglesa con subtítulos también en inglés. El autor detalla que «[1]as convenciones de entrega de este tipo de subtitulado difieren sustancialmente de las seguidas en el subtitulado interlingüístico, y es frecuente ver subtítulos de tres líneas, cargados de repeticiones léxicas o frases inacabadas que son una transcripción literal, palabra por palabra, de los intercambios dialogales» $(2005,18-19)$.

Incluso puede ser determinante el canal de televisión por el que se emita el producto subtitulado en cuestión. Por ejemplo, en su momento TVE aplicaba las siguientes normas, entre las que aquí nos interesa especialmente la primera: subtitular del modo más literal posible, conseguir que el subtítulo aparezca en el mismo momento exacto en el que podemos oír el texto oral y mantener el subtítulo en pantalla el tiempo suficiente para que el espectador lo pueda leer (Izard 2001, 171).

Ahora bien, Mayoral $(2001,41)$ opina que las diferencias entre las lenguas hacen que la traducción literal sea «a veces inconveniente o imposible». A esto se une el hecho de que «el mensaje subtitulado casi siempre debe ser más corto que el original». Pese a ello, «al traductor no le queda más remedio que plegarse a las exigencias del espectador pues si éste percibe la versión subtitulada como mal traducida, este espectador va a experimentar un rechazo hacia el conjunto de la película». Mayoral distingue entre aquellos espectadores con un buen conocimiento de la lengua origen 
y aquellos cuyo conocimiento es escaso o incluso nulo, y señala que, en el primero de los casos, "[l]a versión subtitulada [...] va a ser pues necesariamente un tipo de traducción marcado por la síntesis, por la comunicación de la integridad de los significados y por el literalismo». Respecto a los segundos, afirma que «[n]o ocurre así en casos como España donde el público mayoritario va a apreciar más un estilo más natural con respecto a su lengua y a las leyes generales de la expresión [y que] va a diferenciar menos claramente el subtitulado como la versión traducida». Añade, asimismo, que «[p]aradójicamente, la traducción de subtítulos para espectadores poco conocedores de la lengua original permitirá traducciones que (si logramos abstraernos del destinatario y desde un punto de vista académico) pueden parecer más idóneas que en los otros casos». En resumidas cuentas:

Un mismo producto audiovisual podría ser traducido mediante estrategias muy distintas dependiendo de la situación en la que se produce la traducción. Por ejemplo una película se subtitula con exigencias muy grandes de literalidad cuando el público conoce bien la lengua original y es capaz de seguir la proyección simultáneamente con la banda sonora original y los subtítulos traducidos (película rodada en inglés y proyectada con subtítulos noruegos en Noruega) y por lo tanto de forma automática evalúa la calidad de los subtítulos según sus propios criterios de calidad frente a la situación en que la misma película, con la misma banda sonora original en inglés, se proyecta para un público que no entiende suficientemente la lengua original (el público español por ejemplo) (Mayoral 2013, en línea).

Por tanto, parece claro que son diversos los factores que pueden afectar al grado de literalidad de los subtítulos: el tipo de destinatario, su grado de conocimiento de la lengua origen, el propósito de los subtítulos, el canal de televisión, etc.

\section{LA TRADUCCIÓN LITERAL COMO TÉCNICA O MÉTODO: ¿MÁS FRECUENTE DE LO QUE SE PIENSA?}

Como advertíamos al principio del presente artículo, no es nuestra intención posicionarnos a favor o en contra de la traducción literal, ya sea como método o como técnica. Sin embargo, nos parece relevante plantear una cuestión que una sucinta revisión de distintos trabajos de investigación realizados al respecto pone de manifiesto. Dichos estudios evidencian la abundancia de técnicas literales, pese a la mala prensa que éstas parecen tener en determinados foros.

Reverter recurre a la clasificación de técnicas de Werner Díaz Navarrete (2010) y analiza la versión doblada al español de dos episodios de Los Simpson, prestando especial atención a la traducción de los referentes culturales. Entre sus resultados, destacan los siguientes $(2013,61)$ : 


\begin{tabular}{|c|cc|}
\hline Métodos & $\mathbf{6 0}$ referentes en total & $\mathbf{1 0 0 \%}$ \\
\hline Extranjerizante & 43 & $71,67 \%$ \\
\hline Intermedio & 4 & $6,66 \%$ \\
\hline Familiarizante & 10 & $16,67 \%$ \\
\hline Combinaciones de E y F & 3 & $5 \%$ \\
\hline
\end{tabular}

Tabla 1. Resultados de Reverter

Como se puede observar, casi tres cuartas partes de las técnicas usadas son propias de un método extranjerizante (en terminología de Venuti 1995) o literal (en terminología de Hurtado 2001).

Por su parte, Lozano, tomando como referencia las propuestas de Hurtado (2001), Romero (2003) y Molina (2006), realiza una aproximación a la traducción de la terminología médica, usando como objeto de análisis la versión doblada al español de un episodio de la serie Urgencias. Sus resultados ponen de manifiesto lo siguiente $(2008,71)$ (destacamos en negrita las técnicas más frecuentes):

\begin{tabular}{|c|c|c|}
\hline Técnica & Casos (total 223) & $\%$ \\
\hline Traducción literal & 65 & $\mathbf{2 9 \%}$ \\
\hline Equivalente acuñado & 61 & $\mathbf{2 7 \%}$ \\
\hline Equivalencia inadecuada & 23 & $10 \%$ \\
\hline Compresión lingüística & 21 & $9 \%$ \\
\hline Generalización & 17 & $7 \%$ \\
\hline Transposición & 9 & $4 \%$ \\
\hline Elisión & 9 & $4 \%$ \\
\hline Préstamo & 8 & $2 \%$ \\
\hline Ampliación lingüística & 5 & $1,3 \%$ \\
\hline Particularización & 3 & $0,4 \%$ \\
\hline Compensación & 1 & $0,4 \%$ \\
\hline Amplificación & 1 & $3 \%$ \\
\hline
\end{tabular}

Tabla 2. Resultados de Lozano

De nuevo, podemos observar que la traducción literal, como técnica, es la más numerosa, y que, de hecho, es seguida de cerca por otra técnica de índole literal, como es el equivalente acuñado. 
En su análisis de la serie Modern Family, Pelegrina obtiene las siguientes cifras $(2013,78)$ :

\begin{tabular}{|c|c|c|c|}
\hline \multirow{2}{*}{$\begin{array}{c}\text { Técnicas de traducción } \\
\text { empleadas } \\
\text { en el doblaje }\end{array}$} & Técnicas extranjerizantes & 48 & $\mathbf{5 5 , 2} \%$ \\
\cline { 2 - 4 } & Técnicas intermedias & 20 & $\mathbf{2 3} \%$ \\
\cline { 2 - 4 } & Técnicas familiarizantes & 19 & $21,8 \%$ \\
\hline \multirow{2}{*}{$\begin{array}{c}\text { Técnicas de traducción } \\
\text { empleadas } \\
\text { en la subtitulación }\end{array}$} & Técnicas extranjerizantes & 41 & $\mathbf{4 7 , 7} \%$ \\
\cline { 2 - 4 } & Técnicas intermedias & 29 & 33,7 \\
\cline { 2 - 4 } & Técnicas familiarizantes & 16 & $18,6 \%$ \\
\hline
\end{tabular}

Tabla 3. Resultados de Pelegrina

Un vez más, vemos cómo las técnicas extranjerizantes, ergo cercanas al polo literal (recordemos la figura 2), son las que más abundan, tanto en la versión doblada como en la subtitulada al español.

Por su parte, Martínez Sierra $(2004,413)$, en su estudio sobre la traducción del humor en la serie Los Simpson, indica asimismo resultados como que «[l]a gran cantidad de elementos culturales propios del contexto origen que han permanecido en el texto de la versión meta parece indicar que a la traducción de la serie subyace un planteamiento extranjerizante». Como ya se ha señalado, podemos establecer un claro paralelismo entre método extranjerizante y método literal, por lo que lo descubierto por el autor abre la puerta a la consideración de un alto grado de literalidad en la traducción de la citada serie.

Por último, son de mención obligada los trabajos de Martí Ferriol. Ya en su estudio de 2003 analizaba las técnicas de traducción utilizadas en las versiones doblada y subtitulada al español del filme Monsters' Ball (Marc Forster 2001), y ofrecía los siguientes datos, que desglosa en su tesis doctoral $(2006,111)$ : 


\begin{tabular}{|c|c|c|}
\hline Técnica & Versión doblada & Versión subtitulada \\
\hline Préstamo & 2 & 2 \\
\hline Calco & 1 & 0 \\
\hline Traducción literal & $\mathbf{2 8}$ & $\mathbf{3 8}$ \\
\hline Equivalente acuñado & 3 & 4 \\
\hline Reducción & 11 & 10 \\
\hline Compresión & 0 & 1 \\
\hline Particularizacion & 1 & 0 \\
\hline Generalización & 4 & 2 \\
\hline Transposición & 0 & 0 \\
\hline Descripción & 2 & 0 \\
\hline Ampliación & 5 & 1 \\
\hline Amplificación & 1 & 9 \\
\hline Modulación & 15 & 1 \\
\hline Variación & 2 & 1 \\
\hline Compensación & 0 & 0 \\
\hline Substitución & 0 & 0 \\
\hline Adaptación & 1 & 2 \\
\hline Creación discursiva & 3 & 72 \\
\hline TOTAL & 79 & 1 \\
\hline
\end{tabular}

Tabla 4. Resultados de Martí Ferriol

De nuevo, la técnica de la traducción literal aparece como la más numerosa. A esto, podemos añadir que, en lo que al mantenimiento de los nombres propios (norma identificada por Goris 1993) se refiere, el autor detectó una elevada frecuencia (sobre todo en el caso de la subtitulación al español), materializada por medio del empleo de la técnica de la traducción literal $(2006,65)$.

Datos similares se obtienen si consideramos el resultado de la investigación doctoral de Martí Ferriol, un trabajo en el que llegó a analizar 480 muestras (240 para doblaje y 240 para subtitulación) obtenidas a partir de 5 películas del (sub)género de cine independiente americano. Dichos resultados, en lo que a las técnicas se refiere, se resumen en la siguiente tabla (2006, 273-274): 


\begin{tabular}{|c|c|c|c|c|}
\hline & Técnica & Doblaje & Subtitulación & Total \\
\hline \multirow{6}{*}{ ZE } & Préstamo & 3 & 7 & 10 \\
\hline & Calco & 6 & 8 & 14 \\
\hline & Traducción palabra por palabra & 15 & 21 & 36 \\
\hline & Traducción uno por uno & 18 & 27 & 45 \\
\hline & Traducción literal & 8 & 15 & 23 \\
\hline & Equivalente acuñado & 9 & 15 & 24 \\
\hline \multirow{9}{*}{$\mathrm{Zl}$} & Omisión & 19 & 12 & 31 \\
\hline & Reducción & 54 & 91 & 145 \\
\hline & Compresión & 0 & 0 & 0 \\
\hline & Particularización & 22 & 15 & 37 \\
\hline & Generalización & 14 & 6 & 20 \\
\hline & Transposición & 1 & 1 & 2 \\
\hline & Descripción & 9 & 4 & 13 \\
\hline & Ampliación & 37 & 12 & 49 \\
\hline & Amplificación & 51 & 15 & 66 \\
\hline \multirow{6}{*}{ ZF } & Modulación & 59 & 24 & 83 \\
\hline & Variación & 4 & 4 & 8 \\
\hline & Substitución & 2 & 2 & 4 \\
\hline & Adaptación & 3 & 1 & 4 \\
\hline & Creación discursiva & 84 & 60 & 144 \\
\hline & TOTAL & 418 & 340 & 758 \\
\hline
\end{tabular}

Tabla 5. Resultados de Martí Ferriol

(ZE = zona extranjerizante, $Z I=$ zona intermedia, $Z F=$ zona familiarizante)

Estos resultados son sumamente interesantes, puesto que manifiestan una clara combinación de técnicas propias de todo el abanico de posibilidades en el continuo método literal - método interpretativo-comunicativo que veíamos en la figura 1. Este es un planteamiento que está en consonancia con la afirmación de Mayoral (2013, en línea) de que «[e]n los estudios de traducción se da con demasiada frecuencia el enfoque binario: correcta/incorrecta, cierto/falso, a pesar de que la actividad de la 
traducción [...], no responde a estos juicios binarios sino a criterios de calidad». Así, se podría suponer que, en la práctica real, es común la combinación de técnicas de diferente naturaleza. Estamos, por tanto, ante métodos híbridos, no estancos, que sugieren la idea de la (imprecisa) iusta via media.

Martí Ferriol $(2006,76)$ justifica esta posible mezcla de técnicas propias de métodos distintos del siguiente modo: "Resulta obvio que, aunque el método elegido y sus técnicas asociadas se reproduzcan de forma recurrente a lo largo de todo un texto, se podrán dar momentos concretos en que las restricciones puntuales [...] condicionen el uso de técnicas que no correspondan a priori con la opción metodológica elegida». Mayoral (2013, en línea) se pronuncia en términos similares cuando dice que «[u]na traducción profesional adecuada combinará procedimientos de traducción tanto literales como no literales, será literal y no literal hasta cierto grado y estará muy condicionada por la subjetividad del traductor». A esto añade que «[e]s responsabilidad del traductor utilizar todos los recursos y herramientas disponibles de forma que su traducción sea aceptable y eficaz", un principio que creemos nuestros estudiantes han de conocer.

\section{PALABRAS FINALES}

En cualquier caso, se defienda la postura que se defienda, parece clave saber de qué estamos hablando exactamente en cada momento. Cuando se opina que la traducción literal ha de evitarse sistemáticamente, ¿a qué nos estamos refiriendo? ¿Al seguimiento de un método literal (es decir, de técnicas como el préstamo, el calco, la traducción uno por uno...) o a la aplicación más o menos puntual de la técnica de la traducción literal? ${ }^{6}$ ¿Tenemos claro que, como técnica, no sólo la traducción literal obedece a un método literal, sino que también lo hacen otras técnicas como el préstamo, el calco o, incluso, el equivalente acuñado? Y estas misma preguntas pueden ser aplicables al caso contrario; es decir, si se aboga por la traducción literal como la labor de un traductor para, por ejemplo, una traducción para doblaje.

No se pretende aquí perpetuar el debate traducción literal sí o traducción literal no. Más bien, nuestra intención es la de clarificar conceptos, de modo que, cuando hablemos de traducción literal, en un sentido o en otro, seamos plenamente conscientes de a qué nos estamos refiriendo exactamente. Como docente, me he encontrado en ocasiones en la siguiente situación: un rechazo aprioristico y pleno

6. Como ejemplo de claridad en la argumentación, y sin entrar a valorar su grado de acierto, Mayoral (2013, en línea), por ejemplo, opina que «[l]a defensa de la traducción literal como método de traducción se basa en concepciones anticuadas e inexactas sobre lo que es traducir» y que "[l]os defensores de la traducción literal consideran que existe la traducción correcta y que las demás son incorrectas» (el énfasis en nuestro).

Juan José MARTÍNEZ SIERRA La traducción literal en el ámbito audiovisual. Método y técnica vol. 3-1, June 2017, 13-34

elSSN: 2444-1961

(C) Ediciones Universidad de Salamanca - CC BY-NC-ND 
de la traducción literal por parte de un estudiante ${ }^{7}$. Mi reacción ha sido siempre la misma: tratar de concretar de qué estábamos hablando, con el resultado de que la explicación de la doble naturaleza de la expresión traducción literal, uno como método y dos como técnica, ha resultado realmente útil. Yendo un poco más allá, el asunto se entiende aún mejor si explicamos en clase que los métodos que sigamos no han de ser $100 \%$ estancos, y que los podemos entender como vasos comunicantes o como un continuo en el que las técnicas se reparten, pudiendo puntualmente hacer uso de una que, sobre el papel, pertenece al método opuesto al que estamos asumiendo; por ejemplo, el uso de un préstamo (técnica propia del método de la traducción literal) en una traducción que sigue un método interpretativo-comunicativo (siguiendo la terminología de Hurtado 2001) ${ }^{8}$. De hecho, hemos visto pruebas de que esto es así (véase, por ejemplo, la tabla 5, en la que vemos un reparto de técnicas propias de los dos extremos de la distribución que recoge la figura 1).

Estamos del todo de acuerdo con Mayoral (2013, en línea) cuando expresa que «la traducción literal, como cualquier otra forma de traducir, sí es posible». El autor la entiende aquí como estrategia o técnica de traducción, aunque niega que «siempre constituya la mejor estrategia o técnica». Básicamente, lo que Mayoral hace es reconocer la validez de la traducción literal como técnica, a la vez que precisa que no se trata de una técnica que deba usarse de manera indiscriminada sino sólo en aquellos casos en los que resulte pertinente hacerlo (que son seguramente más de los que pensamos), puesto que habrá otros en los que será mejor recurrir a otro tipo de técnica. No olvidemos que una técnica de traducción literal mal usada puede propiciar un error. La clave reside, seguramente, en la naturalidad de nuestros diálogos traducidos.

Al inicio de este artículo facilitábamos diferentes ejemplos (algunos de ellos, casos erróneos) de traducción literal, y nos preguntábamos cuál era la diferencia entre ellos. Recordemos, por ejemplo, estos dos:

$>$ Human guinea pigs - Cerdos guineanos humanos.

> It's raining cats and dogs - Está lloviendo a cántaros.

7. Este rechazo es también palpable en foros sobre doblaje o subtitulación, por ejemplo, donde abundan las opiniones que critican, sin precisar (¿método o técnica?), la traducción literal y donde parece casi políticamente incorrecto decir que algo se podría haber traducido de manera literal (con el uso de una técnica de índole literal, en este caso). Curiosamente, algunas de estas voces tan críticas con la traducción literal son las mismas que, al mismo tiempo y sin apercibirse de la contradictio in terminis, defienden la virtudes de la extranjerización.

8. Esta consideración está en línea con la afirmación de Mayoral (2013, en línea) de que "[l]as cualidades de la traducción no se pueden describir mediante opciones binarias de cierto / falso, correcto / incorrecto sino que constituyen opciones graduales, de máximo a mínimo».

Juan José MARTÍNEZ SIERRA La traducción literal en el ámbito audiovisual. Método y técnica 
Tras el recorrido realizado, estamos ya en disposición de responder a esa pregunta:

\begin{tabular}{|c|c|c|}
\hline Ejemplo & Método & Técnica \\
\hline $\begin{array}{c}\text { Human guinea pigs }- \\
\text { Cerdos guineanos humanos. }\end{array}$ & Literal & Traducción literal \\
\hline $\begin{array}{c}\text { It's raining cats and dogs }- \\
\text { Está lloviendo a cántaros. }\end{array}$ & Literal $^{9}$ & Equivalente acuñado \\
\hline
\end{tabular}

He ahí la diferencia: mismo método, pero distinta técnica. Por cierto, a nadie le cabrá duda de que, en el primero de los casos, estamos ante un error de traducción, pero seguramente no así en el segundo. Por tanto, si en ambos casos se ha seguido un método literal, la conclusión que procede es que dicho método no constituye una fuente de errores per se, y que posiblemente los errores surgen de su incorrecta aplicación. Recuperamos a continuación otros ejemplos de soluciones traductoras libres de errores puestos al principio de nuestro recorrido:

\begin{tabular}{|c|c|c|c|}
\hline \multicolumn{2}{|c|}{ Ejemplo } & \multirow{2}{*}{ Técnica } & \multirow{2}{*}{ Método } \\
\hline Lengua origen & Lengua meta & & \\
\hline $\begin{array}{l}\text { My computer needs new } \\
\text { software. }\end{array}$ & $\begin{array}{l}\text { Mi ordenador necesita un } \\
\text { nuevo software. }\end{array}$ & Préstamo & \multirow{5}{*}{ Literal } \\
\hline I love science fiction films. & $\begin{array}{l}\text { Me encantan las películas } \\
\text { de ciencia ficción. }\end{array}$ & Calco & \\
\hline The boy eats an apple. & $\begin{array}{l}\text { El chico come una } \\
\text { manzana. }\end{array}$ & $\begin{array}{c}\text { Traducción } \\
\text { palabra por palabra }\end{array}$ & \\
\hline He takes a walk every day. & $\begin{array}{l}\text { Él da un paseo todos los } \\
\text { días. }\end{array}$ & $\begin{array}{l}\text { Traducción } \\
\text { uno por uno }\end{array}$ & \\
\hline $\begin{array}{l}\text { Houston, we have a } \\
\text { problem. }\end{array}$ & $\begin{array}{l}\text { Houston, tenemos un } \\
\text { problema. }\end{array}$ & Traducción literal & \\
\hline
\end{tabular}

Así, y a tenor de lo observado en las distintas investigaciones antes mencionadas, tampoco parece que la traducción literal, como técnica, tenga que, inevitablemente, producir errores cada vez que se aplique, por lo que cabría plantearse si sería procedente que la libráramos de ese halo pernicioso que parece sufrir en determinados foros. Un estudio de caso en este sentido sería bienvenido, a la par que controvertido, habida cuenta de la subjetividad (por no mencionar su perfil prescriptivo) inherente al estudio de las técnicas y de su correcta o incorrecta aplicación.

9. Véase la figura 1, en la que se indica que tanto la técnica de la traducción literal como la del equivalente acuñado se incluyen en el método literal. 
Por otro lado, cabe suponer que merece la pena terminar con esa sensación de que se reduce traducción literal a la técnica, olvidando que existe un método que también se denomina traducción literal (y, seguramente, he aquí el origen del problema, como antes se sugería), así como la confusión terminológica a la que da lugar y que a su vez genera falta de precisión y generalizaciones, quizás, equivocadas.

Creemos que Mayoral (2013, en línea) marca el camino a seguir cuando opina lo siguiente:

Resulta más conveniente por tanto hablar de literalidad, una característica que estará presente en toda traducción de [sic] mayor o menor grado como expresión de una intención de fidelidad a diversos factores presentes en el original (autor, estilo, género) pero que normalmente no prevalece sobre otros factores de situación comunicativa o encargo profesional, dado que la traducción que nos ocupa no es una actividad intelectual ideal sino un proceso comunicativo y una actividad profesional.

Por último, se ataca frecuentemente a la traducción literal (ya sea como método o como técnica), si bien distintas investigaciones, y a los datos nos remitimos, ponen de manifiesto que es un método / técnica (¿paradójicamente?) frecuente en el mundo de la traducción audiovisual. Por tanto, cabe preguntarse si estas críticas están equivocadas o si, por el contrario, lo que ocurre es que, simplemente, se traduce mal. La pregunta, de respuesta nada sencilla, queda en el aire.

\section{REFERENCIAS BIBLIOGRÁFICAS}

BOTELlA TEJERA, Carla. 2007. «Aproximación al estudio del doblaje y la subtitulación desde la perspectiva prescriptivista y la descriptivista: la traducción audiovisual». Tonos 13.

CASTILLO FLORES, César Alejandro. 2011. «Episode twenty three: ¿traducción o adaptación audiovisual?», Babel World. Fecha de acceso 10 de enero de 2016. http://comunidad.uem. es/babel-world/2011/1/12/episode-twenty-three-traduccion-o-adaptacion-audiovisual-.

CHAVES, María José. 2000. La traducción cinematográfica: el doblaje. Huelva: Universidad de Huelva.

CÓMITRE, Isabel. 2009. «Doblaje audiovisual y publicidad. Reflexiones en torno al concepto de manipulación». Translation Journal 13.

DELISLE, Jean. 1993. La traduction raisonnée. Manuel d'initiation à la traduction professionnelle de l'anglais vers le français. Ottawa: Université d'Ottawa.

DELMAS, Christian. 1977. "Les traductions synchrones». En Translating, a Profession. Proceedings of the Eighth World Congress of the International Federation of Translators, ed. por Paul Horguelin. Otawa: Council of translators and interpreters of Canada.

DÍAZ CINTAS, Jorge. 2005. «Nuevos retos y desarrollos en el mundo de la subtitulación». Puentes 6:13-20.

DÍAZ CINTAS, Jorge y Aline REMAEL. 2007. Audiovisual Translation: Subtitling. Mánchester: St. Jerome.

Juan José MARTÍNEZ SIERRA La traducción literal en el ámbito audiovisual. Método y técnica 
FREIJO, Ernesto e Iñaki TORRE. 2014. «El proceso de doblaje». Fecha de acceso 15 de enero. http://www.eldoblaje.com/varios/proceso2.asp.

GORIS, Olivier. 1993. "The Question of French Dubbing: Towards a Frame for Systematic Investigation». Target 5(2): 169-190.

HURTADO, Amparo. 2001. Traducción y traductología. Madrid: Cátedra.

IVARSSON, Jan y Mary CARROLL. 1998. Subtitling. Simrishamm: TransEdit.

IZARD, Natalia. 2001. "La subtitulación para sordos del teletexto en Televisión Española». En Traducción subordinada inglés-español/galego II: el subtitulado, ed. por Lourdes Lorenzo y Ana María Pereira. Vigo: Servicio de Publicacións de la Universidade de Vigo, 169-194.

KAHANE, Eduardo. 1990-91. "Los doblajes cinematográficos. Trucaje lingüístico y verosimilitud». Parallèles 12, 115-120.

KRIEK, Bartho. 2002. "Kijk wie er spreekt. Halve vertalingen in ondertitels». Onze Taal, 276-278.

LABORIS.NET. 2014. «Actor de doblaje». Fecha de acceso 15 de enero de 2016. http://www. laboris.net/static/ca_profesion_actor-doblaje.aspx.

LOZANO, María Dolores. 2008. «Aproximación a la traducción de la terminología médica de series dobladas al Español: el caso de Urgencias». Tesis de máster. Universidad Autónoma de Barcelona.

MARCO, Josep. 2004. El fil d'Ariadna. Anàlisi estilística i traducció literaria. Vic: Eumo.

MARTí FERRIOL, José Luis. 2003. «Estudio descriptivo y comparativo de las normas de traducción en las versiones doblada y subtitulada del filme Monsters' Ball (inglés-español)». Trabajo de investigación. Universitat Jaume I.

MARTí FERRIOL, José Luis. 2006. Estudio empírico y descriptivo del método de traducción para doblaje y subtitulación. Tesis doctoral. Universitat Jaume I.

MARTí FERRIOL, José Luis. 2010. Cine independiente y traducción. Valencia: Tirant lo Blanch.

MARTí FERRIOL, José Luis. 2013. El método de traducción. Doblaje y subtitulación frente a frente. Castellón: Universitat Jaume I.

MARTín, Laurentino. 1994. «Estudio de las diferentes fases del proceso de doblaje». En Transvases culturales: literatura, cine, traducción, ed. por Fernando Eguíluz et al. Vitoria: Universidad del País Vasco, 323-330.

MARTÍNEZ SIERRA, Juan José. 2004. Estudio descriptivo y discursivo de la traducción del humor en textos audiovisuales. El caso de Los Simpson. Tesis doctoral. Universitat Jaume I.

MARTíNEZ TEJERINA, Anjana. 2012. «La integración de los códigos en doblaje: juegos de palabras y restricciones visuales». MonTI 4, 155-180.

MAYORAL, Roberto. 2001. «El espectador y la traducción audiovisual». En La traducción en los medios audiovisuales, ed. por Frederic Chaume y Rosa Agost. Castellón, Universitat Jaume I: Servei de Publicacions, 33-48.

MAYORAL, Roberto. 2013. «La imposibilidad de la traducción literal». Ponencia presentada en el Seminario Internacional científico-práctico Problemas actuales de traducción ruso-español y español ruso, 1-6 de julio de 2013, Universidad de Granada. http://www.ugr.es/ rasensio/ docs/Imposibilidad.pdf.

MOLINA, Lucía. (2006). El otoño del pingüino: análisis descriptivo de la traducción de los culturemas. Castellón: Universitat Jaume I.

NEWMARK, Peter. 1988. A Textbook of Translation. Nueva York: Prentice Hall.

NIDA, Eugene. 1964. Towards a Science of Translating, with Special Reference to Principles and Procedures Involved in Bible Translating. Leiden: E.J. Brill.

PELEGRINA, Zoraida. 2013. «La traducción del humor en el doblaje y en la subtitulación: el caso del personaje de Gloria en la serie Modern Family». Tesis de máster. Universitat Jaume I.

Juan José MARTÍNEZ SIERRA La traducción literal en el ámbito audiovisual. Método y técnica 
PEREIRA, Ana María. 2005. «El subtitulado para sordos: estado de la cuestión en España». Quaderns. Revista de traducció 12, 161-172.

REVERTER, Beatriz. 2013. «La traducción audiovisual de las referencias culturales. El caso de Los Simpson». Proyecto de fin de grado. Universitat de València.

RIOZ, Amaya. 2010. «El doblaje y subtitulado de películas alemanas: efectos para la transmisión lingüística y cultural». Cuadernos de Filología Alemana II, 131-141.

ROMERO, María Guadalupe. 2003. "Un estudio descriptivo sobre la traducción de los dialectos geográfico-sociales del italiano al español en el ámbito audiovisual: doblaje y subtitulación: la traducción de II Positano». Trabajo de investigación. Universitat Autònoma de Barcelona.

SIMÓN, Ana María. 2008. «Adaptar y dirigir el doblaje de Los Simpson». Quaderns de Filologia. Estudis Literaris XIII, 21-23.

SORROCHE, Sebastián. 2010. «El perfil del traductor audiovisual y de videojuegos: una mirada al mercado». La linterna del traductor 4.

TORREGROSA, Santiago. 2012. «El viaje del sobre acolchado. Veinte años de subtitulado electrónico (1990-2010)». En Reflexiones sobre la traducción audiovisual. Tres espectros, tres momentos, ed. por Juan José Martínez Sierra. Valencia: Universitat de València, 81-92.

TOURY, Gideon. 1995. Descriptive Translation Studies — and Beyond. Ámsterdam / Filadelfia: John Benjamins.

VÁZQUEZ AYORA, Gerardo. 1977. Introducción a la traductología. Curso básico de traducción. Washington D.C.: Georgetown University Press.

VENUTI, Lawrence. 1995. The Translator's Invisibility. Londres / Nueva York: Routledge.

VINAY, Jean-Paul y Jean DARBELNET. 1958. Stylistique comparée du français et de l'anglais. Montreal: Beauchemin.

WERNER DÍAZ NAVARRETE, Marleen. 2010. «Un estudio traductológico de los referentes culturales extralingüísticos en la subtitulación: Ejemplificados en la serie de televisión Cuéntame có mo pasó». Tesina. Copenhagen Business School.

WHITMAN, Candace. 1992. Through the Dubbing Glass. The Synchronization of American Motion Pictures into German, French and Spanish. Frankfurt am Main: Peter Lang. 